\title{
Control de mérito en la Acción Extraordinaria de Protección
}

Fact check in the Extraordinary Protection Action

\section{Josuha Cisneros Rodríguez*}

Investigador Jurídico Independiente

Información del Artículo

Original - Ruptura, 2020

Artículo recibido/ Received: 3 de octubre, 2020

Artículo aceptado/Accepted: 9 de noviembre, 2020

\section{Citación}

Cisneros, J. (2020). Control de mérito en la Acción Extraordinaria de Protección. Revista Ruptura de la Asociación Escuela de Derecho PUCE. Edición 2020, p (211-225).

DOI: $10.26807 /$ rr.vi02.29

Resumen: El objeto de esta investigación es abordar uno de los puntos más polémicos de la acción extraordinaria de protección: el control de mérito. Se trata, pues, de la posibilidad de que la Corte Constitucional pueda revisar los hechos que motivaron la controversia. Sin duda alguna la acción extraordinaria de protección es la garantía jurisdiccional más usada en el país y sobre la cual más jurisprudencia se ha desarrollado. En tal sentido, la Corte de 2019 ha cambiado de argumentos y de línea jurisprudencial en este tema, por lo que el presente estudio analizará esta modificación, y alguna inconsistencia o contradicción de la misma.

* Estudiante de sexto semestre de Derecho en la Universidad Nacional de Chimborazo, Riobamba - Ecuador. Correo electrónico: josuha.cisneros@gmail.com 
Palabras clave: Acción extraordinaria de protección, control de mérito, Constitución, Corte Constitucional, garantía, jurisprudencia.

\begin{abstract}
This research will study the "fact check" in the extraordinary proteccion action. This means that the Constitutional Court could review the facts of the original case. The extraordinary proteccion action is the most used jurisdictional guarantee in the country. That is why the Court has developed a great deal of jurisprudence on this. Without doubt this review of facts is a controversial side of this guarantee. Recently, the Court has changed arguments on this issue. So this investigation will analyze these changes and some apparent contradictions.
\end{abstract}

Keyword: Extraordinary protection action, fact check, Constitution, Constitutional Court, guarantee, jurisprudence.

\title{
Introducción
}

La acción extraordinaria de protección significó un cambio basilar en la concepción del régimen de garantías jurisdiccionales determinado en la Constitución de 1998. Esta nueva garantía abrió la puerta al control de constitucionalidad de las actuaciones judiciales, toda vez que se reconoce la posibilidad de que las decisiones tomadas por los jueces puedan violar derechos constitucionales.

Nos encontramos frente a la garantía jurisdiccional más utilizada en el país, según el Informe de Rendición de Cuentas de la Corte Constitucional (2020), durante el año 2019 se receptaron 6.698 causas, de las cuales 3.311 fueron acciones extraordinarias de protección, es decir, el 49,43\% de casos que llegaron en dicho año a la Magistratura pertenecen a esta categoría. (p. 16).

Asimismo, durante alrededor de once años, la jurisprudencia sobre esta acción ha sido abundante y en ocasiones, como diría Oyarte, farragosa. Desde el primer fallo dictado por la Corte Constitucional para el periodo de transición -Sentencia No. 001-09-SEP-CC-, hasta los dictámenes más recientes -Sentencias No. 1000-17-EP/20 y No. 
1828-15-EP/20-, es indiscutible que se trata de la garantía de mayor desarrollo jurisprudencial en el país.

Por los motivos antes mencionados, la demanda de protección extraordinaria es un tema de obligatorio conocimiento para todo profesional del derecho, pues resulta muy probable que, al menos una vez en el ejercicio de la profesión, nos encontremos frente a esta garantía. En este sentido, han sido varias las críticas de jueces constitucionales sobre el uso que los abogados han hecho de ella, se los acusa de valerse de tal acción como una suerte de cuarta instancia y, en consecuencia, de congestionar el trámite de las causas en la Corte Constitucional.

Los problemas jurídicos que han surgido en la práctica de esta garantía son varios; las interpretaciones constitucionales han solucionado algunos, han empeorado otros, y han generado otros tantos nuevos. Sin embargo, el estudio integral de la tornadiza jurisprudencia constitucional ayuda, en retrospectiva, a reconocer estos conflictos.

Por tal razón esta investigación plantea un estudio de la jurisprudencia constitucional, esto específicamente en cuanto a la posibilidad de analizar los hechos del caso subyacente en una acción extraordinaria de protección, a propósito del reciente cambio de criterio por parte de la Corte de 2019.

\section{La acción extraordinaria de protección y un acercamiento al control de mérito}

El sistema de garantías jurisdiccionales instaurado por la Constitución de 2008 procuró cambios significativos en la protección de derechos y en la visión del constitucionalismo ecuatoriano. Indudablemente, con la Constitución de 1998 resulta impensable la posibilidad de acudir a la justicia constitucional para objetar providencias judiciales (Oyarte, 2020).

Una de las innovaciones de la Constitución de 2008 fue, precisamente, la acción extraordinaria de protección. Esta garantía tiene 
por objeto, como su nombre lo dice, la protección de derechos constitucionales, pero solamente cuando estos hayan sido vulnerados en el ejercicio de la potestad jurisdiccional a través de sentencias, autos definitivos y resoluciones con fuerza de sentencia o, si los fallos se expiden violando el debido proceso (CRE, 2008).

Siguiendo esta línea, la Corte Constitucional (2019) en la Sentencia No. 1534-14-EP/19 ha precisado que este organismo "puede verificar (...) que la decisión impugnada corresponda al tipo de decisiones antes mencionadas, es decir que esté dentro del ámbito material de sus competencias" (párr. 9). Según ha señalado la propia Corte (2019) en la Sentencia No. 154-12-EP/19, esto sería posible incluso a pesar de que la causa se haya admitido a trámite, de modo que:

(...) si en la etapa de sustanciación el Pleno de la Corte identifica, de oficio, que el acto impugnado no sea una sentencia, un auto definitivo o una resolución con fuerza de sentencia (...), la Corte no puede verse obligada a pronunciarse sobre el mérito del caso. (párr. 52)

Entonces, queda claro cuales son las decisiones jurisdiccionales que son objeto de la demanda de protección extraordinaria; sin embargo, de acuerdo con la jurisprudencia emitida por la Corte Constitucional, existe la posibilidad de realizar un "control de mérito", es decir, un análisis integral del fondo de la causa, lo que se traduce en una dilatación del ámbito de actuación de la justicia constitucional.

En este sentido, la jurisprudencia constitucional ecuatoriana ha esgrimido tres razonamientos con el fin de legitimar dicho análisis de fondo: (i) la protección de derechos; (ii) el principio iura novit curia; y; (iii) la dimensión objetiva de la acción extraordinaria de protección (Morales, 2019). Estos argumentos han sido usados de manera habitual y conjunta para justificar el control de mérito; empero, la Corte Constitucional de 2019 ha superado estas interpretaciones y ha establecido una nueva línea jurisprudencial que se estudiará en apartados posteriores. 


\subsection{La naturaleza de la acción extraordinaria de protección}

El constituyente ha incurrido en contradicciones sobre esta garantía jurisdiccional, toda vez que el artículo 94 de la Constitución la denomina "acción" y "recurso" a la vez. A fin de resolver el aparente conflicto es necesario recurrir a la doctrina. Couture (1958) afirma que "la acción es, (...), el poder jurídico que tiene todo sujeto de derecho, de acudir a los órganos jurisdiccionales para reclamarles la satisfacción de una pretensión" (p. 57); entonces, la acción es la facultad de activar el sistema jurisdiccional. Por otra parte, el recurso, de acuerdo con el mismo autor, "quiere decir, literalmente, regreso al punto de partida" (p. 340), lo que implica que ya se ha activado el aparato jurisdiccional, por lo tanto, un recurso se usa como "medio de impugnación por virtud del cual se recorre el proceso” (p. 340).

Ahora bien, a primera vista la acción extraordinaria de protección parece ser un recurso puesto que se objeta una decisión judicial, entendiéndose que ya se ha activado el sistema jurisdiccional mediante el fallo objetado, pero en realidad se trata de una acción, toda vez que a través de ella "se conforma un proceso autónomo posterior al que se impugna” (Oyarte, 2020, p. 40).

En la misma línea, la Corte Constitucional (2011) en la Sentencia No. 013-11-SEP-CC señala que:

(...) el accionante (...) confunde a la Corte Constitucional con un Tribunal de Alzada (...). Pretende que esta Corte se inmiscuya revisando el fondo del asunto, sin tomar en cuenta que el juez constitucional no puede suplantar al juez natural en el análisis de los asuntos fácticos o de mera legalidad, pues la Corte Constitucional no es una instancia judicial de análisis sobre la litis, sino que su competencia radica exclusivamente en este tipo de acciones, en examinar la conformidad y observancia del trámite, y que en la misma no se violen derechos. (p.12)

Entonces, queda claro que la acción extraordinaria de protección es un proceso independiente que activa y se desarrolla en la justicia 
constitucional, por lo tanto, su naturaleza es la de una acción, mas no la de un recurso ni mucho menos se trata de una cuarta instancia.

\section{Breve reseña del argumento: protección de derechos}

La Corte Constitucional en transición (2009) inicia con la concepción de esta tesis en el voto salvado de la Sentencia No. 019-09-SEPCC, el juez constitucional analiza el fondo del caso subyacente, concluyendo que "es evidente que el IESS ha vulnerado el derecho al habeas data del peticionario" (p. 38); así, el juez infiere que una institución pública no judicial -IESS- ha violado derechos, aspecto que excedería el alcance de la garantía en cuestión. Además, el magistrado sugiere que el Tribunal debería ordenar al IESS que "implemente mecanismos efectivos de acceso a la información por parte de los afiliados (...) para evitar la vulneración de derechos fundamentales, bajo los parámetros establecidos en la Constitución de la República y esta sentencia” (p. 39). De tal modo surge la idea de que la Corte use el argumento "proteger derechos" con el objeto de legitimar un control de mérito.

Posteriormente la Magistratura ha usado este argumento en sus sentencias. Morales (2019) asegura que "se han encontrado 40 decisiones en que la Corte Constitucional deja en firme una decisión, luego de ejecutar un examen sobre el fondo de la controversia. Estos dictámenes aumentan progresivamente desde el 2009 hasta el 2013" (p. 18).

\section{Breve reseña del argumento: iura novit curia}

La competencia para conocer la acción extraordinaria de protección recae sobre el magistrado constitucional; para Oyarte (2020) el juez, en virtud del principio iura novit curia, puede "fundamentar su decisión en cualquier precepto constitucional vulnerado, aunque no se lo invoque expresamente $y,(.$.$) aunque el peticionario lo haya$ fundamento en uno distinto o incluso equivocado" (p. 65). Asimismo, la Corte Constitucional (2010) en la Sentencia No. 0010-10-SEPCC señaló que, en función de la regla iura novit curia, "esta Corte está 
plenamente facultada para analizar y pronunciarse sobre una serie de aspectos no argüidos por las partes y que podrían devenir en vulneraciones a derechos constitucionales" (p.15).

Para resumir, la regla iura novit curia es un principio procesal del derecho constitucional, en razón del cual, los operadores de justicia pueden aplicar una norma distinta a la solicitada por los participantes de un proceso constitucional (LOGJCC, 2009, art. 4 numeral 13).

Ahora bien, la Magistratura superó el argumento de la protección de derechos para arribar a este principio; en tal sentido, la Corte Constitucional (2018) ha manifestado en la Sentencia No. 003-18-SEP-CC que, "en atención a (...) los principios iura novit curia, (...) esta Corte está facultada para analizar la integralidad del proceso y la posible afectación a derechos constitucionales cuando los operadores de justicia de instancia no lo hubieren realizado" (p. 19). Sobre el tema, Morales (2019) señala que:

(...) la Corte Constitucional no ha utilizado mucho el fundamento del iura novit curia para dejar en firme una sentencia, luego de analizar la sustancia del caso. De esta manera, de las 636 sentencias revisadas se detectaron 13 decisiones de este tipo que surgieron a partir del año 2015. (p. 24)

\section{La "dimensión objetiva" de la acción extraordinaria de protección: una interpretación superada por la Corte de 2019}

Como ya se ha mencionado a lo largo de esta investigación, la jurisprudencia constitucional acerca de este tema ha sido contradictoria, inicialmente se restringió la posibilidad de que la Magistratura se pronuncie sobre el fondo de la controversia; sin embargo, también se ha justificado el control de mérito con la protección de derecho y el principio iura novit curia. La jurisprudencia trascendió estos dos argumentos con otro tanto más controvertido: la "dimensión objetiva".

La Corte Constitucional (2014) en el fallo No. 113-14-SEP-CC plantea la "dimensión objetiva" de esta garantía jurisdiccional como nuevo 
argumento para el control de mérito. La Magistratura en dicha sentencia expone que "(...) asumirá también la dimensión objetiva de la acción extraordinaria de protección y establecerá un precedente en la materia" (p. 10). Dejando de lado lo cuestionable del razonamiento y de los argumentos de la Corte que fundamentan esta decisión, resulta tanto o más criticable que no se define la supuesta dimensión objetiva, mucho menos se la demarca en cuanto a sus alcances y restricciones, simplemente se limita a "asumirla".

En posteriores fallos la Corte se pronuncia sobre la visión del constitucionalismo ecuatoriano en torno a esta garantía jurisdiccional, ahora sí, precisando a que alude la presunta bidimensionalidad ${ }^{1}$ de la acción extraordinaria de protección. La Magistratura ha explicado que la dimensión subjetiva responde a la tutela de derechos vulnerados por sentencias, mientras que la dimensión objetiva tiene relación con la disposición de precedentes jurisprudenciales en esquemas fácticos específicos (CCE-EP-119-15-SEP-CC, 2015). Con esta explicación, por una parte parece aclararse el panorama en cuanto a la dimensión objetiva, al menos respecto a su definición, sin embargo, sobran interrogantes.

La Corte Constitucional de 2019 se ha desvinculado de dicha línea jurisprudencial, y hasta cierto punto parece criticarla. El Tribunal considera que no existe más "dimensión" que la posibilidad de proponer esta acción ante sentencias judiciales que hayan vulnerado derechos; sin embargo, la propia Magistratura determina que ocurren casos cuya gravedad exige un pronunciamiento de la Corte sobre posibles violaciones de derechos en el fondo del asunto. Por lo tanto, se acepta la posibilidad de un análisis de mérito; empero, se niega la existencia de una doble dimensión. Continuando con el argumento, la Corte (2019) en la Sentencia No. 1162-12-EP/19 expone que “(...) solo de manera excepcional se puede ampliar su ámbito de actuación y analizar los hechos o procedimientos administrativos a los que se

1 Se hace referencia a una doble dimensión porque al asumir que la acción extraordinaria de protección cuenta con una dimensión objetiva, implícitamente se reconoce la existencia de otra subjetiva. 
refieran esas decisiones jurisdiccionales" (párr. 61), razón por la cual no debe tomarse como regla general un estudio de la sustancia de la causa, a contrario sensu, esta es una facultad inusitada.

Asimismo, la Corte sostiene que el fundamento del examen fáctico radica en su carácter de máximo órgano de control, interpretación y administración de justicia constitucional, motivo por el cual está obligada a verificar que las garantías jurisdiccionales cumplan con su fin, cuestión que en algunos casos podría exigir que el Tribunal examine los hechos del caso subyacente (CCE-EP-1162-12-EP/19, 2019). Con base en esta argumentación, la Magistratura se aparta de la dimensión objetiva planteada en la jurisprudencia previa $\mathrm{y}$, aunque permite el control de mérito, lo hace en función de otro contexto interpretativo.

En el mismo sentido, la excepcionalidad sólo podría ser verificada de oficio (CCE-EP-176-14-EP/19, 2019) y en el contexto de casos que provengan de sentencias emitidas en el marco de garantías jurisdiccionales, en las cuales los jueces ordinarios actúan en virtud de la competencia constitucional (CCE-EP-162-12-EP/19, 2019). Esto implica la prohibición de un análisis fáctico si el fallo que origina la acción extraordinaria de protección emana de la justicia ordinaria, ya que no se trata una cuestión de orden constitucional, sino meramente legal. Vale enfatizar esta idea, el control de mérito procede exclusivamente cuando se objeta una sentencia dictada por la justicia constitucional.

Posteriormente la Corte (2019) en la Sentencia 1162-12-EP/19 delimita las condiciones que se deben cumplir para aplicar el análisis de fondo, entonces se debe verificar:

(i) que la autoridad judicial inferior haya violado el debido proceso $\mathrm{u}$ otros derechos de las partes en el fallo impugnado o durante la prosecución del juicio (...); (ii) que prima facie, los hechos que dieron lugar al proceso originario puedan constituir una vulneración de derechos que no fueron tutelados por la autoridad judicial inferior; y, (iii) que el caso no haya sido seleccionado por esta Corte para su revisión. (p. 55) 
Adicionalmente, la Magistratura señala otro requisito, de modo que la causa debe cumplir al menos uno de los siguientes cuatro criterios de relevancia: gravedad del asunto; novedad del caso; relevancia nacional; o, la inobservancia de precedentes establecidos por la Corte (CCE-EP-176-14-EP/19, 2019).

Ahora, es pertinente realizar un análisis de la aplicación material de la nueva línea jurisprudencial que regula el control de mérito; en este sentido, la Corte Constitucional (2020) en la Sentencia No. 1000-17-EP/20 realiza un examen de los hechos del caso originario, decretando que una autoridad no judicial -Registro Civil- incurrió en vulneraciones de derechos del accionante.

La citada providencia inicia, igual que todas las causas de acción extraordinaria de protección, verificando la violación de derechos constitucionales proveniente del fallo objetado, siendo el caso que dicha sentencia vulnera el derecho a la seguridad jurídica y el debido proceso en la garantía de la motivación. Posteriormente, antes de emprender un control de mérito, se comprueba que la causa proviene de la justicia constitucional -acción de protección-; y, como ya se ha verificado que la autoridad judicial transgredió derechos del accionante, se cumple el primer filtro. A continuación se constata que, prima facie, los hechos objeto de la controversia podrían implicar detrimento de derechos no tutelados por los jueces de instancia. Asimismo, se inspecciona que la causa no haya sido seleccionada por la Magistratura para su revisión (CCE-EP-1000-17-EP/20, 2020).

Adicionalmente, como ya se ha mencionado en párrafos previos, debe cumplirse al menos uno de los cuatro presupuestos necesarios para el análisis de fondo: gravedad de la cuestión; novedad del asunto; relevancia nacional; o, la inobservancia de precedentes constitucionales. En el antedicho dictamen se cumple la gravedad del caso, toda vez que la condición de vulnerabilidad del sujeto -adulto mayor- involucra la necesidad de proporcionar amparo eficaz e inmediato al sujeto con el fin de evitar que el daño sea irremediable. (CCE-EP-1000-17-EP/20, 2020). 
Por último, el Tribunal Constitucional se plantea las implicaciones procesales de realizar un control de mérito. Como primer punto, se reconoce que las partes procesales de la demanda de protección extraordinaria y del proceso originario no son las mismas. Por esta razón, es preciso que la contraparte de instancia -Registro Civil- sea tratada como parte procesal en la causa constitucional, en tal sentido, las correspondientes notificaciones son obligatorias. Como segunda medida, la Corte advierte la necesidad de efectuar una nueva audiencia con la autoridad judicial accionada y la parte procesal del caso subyacente, con el objetivo de analizar el marco fáctico de dicho proceso.

Con estos antecedentes, la Magistratura procede con el control de mérito, lo que lleva a la Corte a preguntarse si existió vulneración de derechos en el fondo de la controversia, ya sea por parte de de un particular o de una autoridad pública no judicial. El Tribunal Constitucional concluye que la negligencia del Registro Civil, efectivamente, se tradujo en violación del derecho a obtener servicios públicos de calidad y del derecho a la identidad personal del accionante.

De modo complementario, Agustín Grijalva Jiménez y Daniela Salazar Marín, magistrados de la Corte Constitucional, desarrollaron votos concurrentes en los que, de manera interesante, realizan un control de mérito más amplio que el efectuado en la propia sentencia. En este aspecto, ambos jueces coinciden en que los hechos del caso denotan la vulneración del derecho a la personalidad jurídica; sin embargo, la jueza constitucional Daniela Salazar también plantea argumentos que, a su criterio, señalan la existencia de una violación del derecho al debido proceso en las garantías de defensa y presunción de inocencia.

En definitiva, la Corte Constitucional ha erigido una nueva línea jurisprudencial, misma que consolida indicadores más idóneos para la aplicación del control de mérito, lo que otrora era inexistente. Sin embargo, se advierte una aparente o real contradicción en cuanto un aspecto referido en esta novel jurisprudencia.

La propia Corte Constitucional (2019) en la sentencia No. 116212-EP/19 apunta que el control de mérito solo podría verificarse de 
oficio, de igual forma en el fallo No. 176-14-EP/19 precisa el mismo punto, señalando que la ampliación del ámbito de actuación del Tribunal a revisar lo decidido por los jueces de instancia en procesos constitucionales no se realiza a petición de parte.

Esta cuestión parece clara; no obstante, la misma Corte (2019) en el fallo No. 176-14-EP/19 del juez constitucional Enrique Herrería expresa que:

(...) en la acción extraordinaria de protección le corresponde limitarse a verificar las vulneraciones del debido proceso o de otros derechos constitucionales por parte de la autoridad judicial. Esto, al (sic) menos que el accionante haya solicitado un control de méritos, en cuyo caso la Corte verificará si se cumplen los presupuestos para determinar la excepcionalidad del caso. (párr. 65)

El primer criterio señala que el control de mérito no se realiza a petición de parte, lo que implicaría que la Corte no está obligada a motivar la negativa a esta solicitud; por el contrario, el otro argumento faculta al accionante a solicitar el examen de fondo, de lo que se deduce que la Magistratura estaría forzada a pronunciarse sobre la excepcionalidad del caso para no caer en inmotivación.

El problema surge con la incertidumbre del accionante y el amplio margen de discrecionalidad y arbitrariedad de los jueces constitucionales para pronunciarse o no sobre la verificación de la excepcionalidad del caso. Concretamente, cuando el demandante solicite un análisis de fondo, se desconoce qué postura tomará el juez constitucional, este bien podría argumentar que dicho examen fáctico se ejecuta únicamente de oficio, negándose a motivar tal decisión. Esta posibilidad es totalmente contradictoria con la disposición del juez Herrería que, en el mismo fallo, indica que el control de mérito se realiza a petición de parte. 


\section{Conclusiones}

La Corte Constitucional ha permitido analizar la sustancia del caso en la acción extraordinaria de protección, cuestión ampliamente criticada desde sus inicios; en este sentido, la calidad de los argumentos, la desnaturalización de esta garantía, la vaguedad de sus límites y alcances, y la actuación de la Corte como juez de instancia han sido algunas de las posturas críticas ante la posibilidad que ha brindado la Magistratura.

A pesar de ello, la nueva línea jurisprudencial marcada por la Magistratura de 2019 ha determinado nuevos fundamentos para el control de mérito, además ha limitado el ejercicio de esta facultad a casos excepcionales que cumplan requisitos específicos previamente determinados.

Aunque la posibilidad de realizar un control de mérito en una acción extraordinaria de protección no está prevista en la Constitución del Ecuador, es la Corte Constitucional quien, como máximo organismo de interpretación y justicia constitucional, ha expresado que existen casos en los que la necesidad de asegurar que las garantías jurisdiccionales cumplan con sus objetivos constitucionales exige un pronunciamiento sobre los hechos que dieron origen al proceso constitucional de instancia.

Sin embargo, no faltan quienes consideran que la Corte Constitucional debe respetar la naturaleza y alcance de las garantías jurisdiccionales; razón por la cual permitir un control de mérito en una acción extraordinaria de protección sería un criterio hipergarantista que no justifica una extralimitación de la Corte Constitucional, ni siquiera cuando se pretenda tutelar derechos constitucionales. 


\section{Referencias Bibliográficas}

Asamblea Nacional del Ecuador. Ley Orgánica de Garantías Jurisdiccionales y Control Constitucional. [LOGJCC]. (21 de septiembre de 2009). RO Suplemento 52 de 22 de octubre de 2009)

Constitución de la República del Ecuador [Const]. 2008. 2da Ed. CEP.

Couture, E. J. (1958). Fundamentos del Derecho Procesal Civil. Buenos Aires: Depalma. Recuperado de https://www.upg.mx/wp-content/ uploads/2015/10/LIBRO-42-Fundamentos-de-Derecho-Procesal-Civil.pdf

Morales, A. (2019). La desnaturalización de la acción extraordinaria de protección a partir de la jurisprudencia de la Corte Constitucional. Quito: Universidad San Francisco de Quito. Recuperado de: http://repositorio. usfq.edu.ec/bitstream/23000/8197/1/142339.pdf

Oyarte, R. (2020). Acción Extraordinaria de Protección. Quito: Corporación de Estudios y Publicaciones.

\section{Jurisprudencia Constitucional}

Corte Constitucional del Ecuador. Sentencia No. 013-11-SEP-CC, Caso No. 0793-09-EP de 18 de agosto de 2011. Juez ponente: Hernando Morales Vinueza. R.O. No. 629 de 30 de enero de 2012

Corte Constitucional del Ecuador. Sentencia No. 0010-10-SEP-CC, Caso No. 0502-09-EP de 08 de abril de 2010. Juez ponente: Luis Jaramillo Gavilanes. R.O. No. 177 de 22 de abril de 2010.

Corte Constitucional del Ecuador. Sentencia No. 019-09-SEP-CC, Caso No. 0014-09-EP de 06 de agosto del 2009. Jueza ponente: Nina Pacari Vega.

Corte Constitucional del Ecuador. Sentencia No. 113-14-SEP-CC, Caso No. 0731-10-EP de 30 de julio de 2014. Jueza ponente: Ruth Seni Pinoargote. 
Corte Constitucional del Ecuador. . Sentencia No. 119-15-SEP-CC, Caso No. 0537-11-EP de 22 de abril de 2015. Juez ponente: Marcelo Jaramillo Villa.

Corte Constitucional del Ecuador. Sentencia No. 003-18-SEP-CC, Caso No. 0948-13-EP de 03 de enero de 2018. Jueza ponente: Pamela Martínez Loayza.

Corte Constitucional del Ecuador. Sentencia No. 1162-12-EP/19, Caso No. 1162-12-EP de 02 de octubre de 2019. Jueza ponente: Daniela Salazar Marín.

Corte Constitucional del Ecuador. Sentencia No. 176-14-EP/19, Caso No. 17614-EP de 16 de octubre de 2019. Juez ponente: Enrique Herrería Bonet.

Corte Constitucional del Ecuador. Sentencia No. 1000-17-EP/20, Caso No. 1000-17-EP de 23 de septiembre de 2020. Jueza ponente: Carmen Corral Ponce.

Corte Constitucional del Ecuador. Sentencia No. 154-12-EP/19, Caso No. 15412-EP de 20 de agosto de 2019. Jueza ponente: Daniela Salazar Marín.

Corte Constitucional del Ecuador. Sentencia No. 1534-14-EP/19, Caso No. 1534-14-EP de 16 de octubre de 2019. Juez ponente: Alí Lozada Prado.

Corte Constitucional del Ecuador (2020). Informe de Rendición de Cuentas 2019. Recuperado de https://www.corteconstitucional.gob.ec/index. $\mathrm{php} /$ rendicion-de-cuentas/rdc-2018-2030/4100-informe-de-rendici\%C3\%B3n-de-cuentas-2019/file.html 